\title{
Flexible Integration of Alternative Energy Sources for Autonomous Sensing
}

\author{
Alex S. Weddell, Neil J. Grabham, Nick R. Harris, Neil M. White \\ Pervasive Systems Centre, School of Electronics and Computer Science, University of Southampton \\ Southampton, U.K. SO17 1BJ \\ \{asw05r, njg, nrh, nmw\}@ecs.soton.ac.uk, Tel. +44 (0)23 8059 4996, Fax. +44 (0)23 80592901
}

\begin{abstract}
Recent developments in energy harvesting and autonomous sensing mean that it is now possible to power sensors solely from energy harvested from the environment. Clearly this is dependent on sufficient environmental energy being present. The range of feasible environments for operation can be extended by combining multiple energy sources on a sensor node. The effective monitoring of their energy resources is also important to deliver sustained and effective operation.

This paper outlines the issues concerned with combining and managing multiple energy sources on sensor nodes. This problem is approached from both a hardware and embedded software viewpoint. A complete system is described in which energy is harvested from both light and vibration, stored in a common energy store, and interrogated and managed by the node.
\end{abstract}

\section{Introduction}

Wireless autonomous sensors are devices with sensing, processing and wireless communication capabilities. In order to deliver truly autonomous operation, sensor nodes must not depend on an external power source. Conventionally, wireless sensing devices are powered by non-rechargeable batteries which must be replaced when depleted (imposing a maintenance requirement that can prove costly during long deployments), or must support the operation of the node for its complete lifetime.

For sustained operation, it follows that nodes should not be reliant on non-rechargeable batteries, but instead generate their own energy in-situ. Energy harvesting is a developing technology area, and prominent technologies facilitate the generation of electricity from light (photovoltaics), vibration (vibration energy harvesting), or thermal gradients (thermoelectrics). The intermittent nature of many environmental energy sources means that viable devices must harvest energy from their operating environment when possible, and buffer excess energy in supercapacitors [1].

In general, harvested energy is scarce and often unpredictable. The range of feasible environments for operation can be extended by combining multiple energy harvesting sources on one node, and managing energy resources carefully. In this paper we present, as an example, a system incorporating photovoltaic and vibration energy harvesting. Algorithms to manage these energy resources are introduced, and the embedded software is developed in line with a defined hardwaresoftware architecture [2].
The embedded software structure implemented is adaptable to deal with a range of energy sources and stores, and facilitates the monitoring of energy resources in order to deliver sustained operation for the autonomous sensor. Future developments to the system are considered, including plans for monitoring non-trivial parameters such as the open-circuit voltage of inputs to the system in order that the expected maximum power can be estimated.

The structure of the paper is as follows. Firstly, we consider the behaviour of conventional energy sources, then we introduce the characteristics of energy harvesting devices, and outline options for energy storage devices. In the second part of this paper, the behaviour of the complete system is considered, with detail given about the embedded software and the hardware structure.

\section{Conventional Energy Sources}

Generally, autonomous sensors are powered by either alkaline or long-life lithium batteries. Alkaline cells have a relatively low cost, but also a moderate capacity and shelf life of a few years. Long-life lithium cells have a higher energy density, and longer shelf life of around 10 years, but are more costly and have other additional drawbacks regarding safety and disposal [1].

A key consideration for battery-powered systems is the amount of power used over their lifetime, and the impact of battery replacement. The fact that these conventional power sources cannot be recharged in-situ means that they are reliant on outside maintenance, and designs must have a substantial margin of error built-in to deliver confidence that systems will operate for their designed lifetime. The state-of-charge of long-life battery types is difficult to determine, and this means that there can be a relatively short time (in which the battery can be replaced without adverse impact) between the system detecting the end-oflife of the battery, and its complete failure [3].

It must, however, be noted that a major advantage of batteries is their reliability. While their performance is affected somewhat by their operating temperature, they are not reliant on environmental conditions to generate their energy (unlike energy harvesting devices). Their performance is relatively well understood, and they are a proven technology which does not require complex energy conditioning circuitry.

\section{Energy Harvesting Devices}

As described earlier, energy can be harvested primarily from ambient light, vibration, and thermal gradients. It is also possible to transfer energy wirelessly to nodes by means of effects such as electromagnetic induction (as used by RF-ID tags), but this type of energy transfer is beyond the scope of this paper. 
While successful implementations of photovoltaic and vibration energy harvesting systems have been reported in the literature [4][5], the requirement for a large and sustained thermal gradient across the device has meant that few practical deployments of thermoelectric generators have been possible to date. Research developments are improving the efficiency of thermoelectric generators and it is likely that a larger number of implementations will become feasible in the near future [6].

A well-lit office will have an average light level of around 500 lux. In this setting, an amorphous silicon indoor photovoltaic cell of $64 \mathrm{~cm}^{2}$ will provide a maximum DC power output of $800 \mu \mathrm{W}$ at around $3.3 \mathrm{~V}$ [1]. In situations where lights are extinguished at night, this energy level will clearly only apply during the day.

Publicity from PMG Perpetuum indicates that its commercially-available PMG17 vibration energy harvester can generate at least $500 \mu \mathrm{W}$ on $80 \%$ of machines, and at least $1.0 \mathrm{~mW}$ on $60 \%$ of machines [7]. While these levels are derived from real deployments, they can only apply when the machinery is operational.

Micropelt's TE-Power-One is a thermoelectric generator module with attachable heat sink and power conditioning circuitry. With a hot-side temperature of $50^{\circ} \mathrm{C}$ and an ambient temperature of $25^{\circ} \mathrm{C}$, their generator can be expected to produce $300 \mu \mathrm{W}$ (before conditioning) at approximately $0.3 \mathrm{~V}[8]$ - a problematically small DC voltage.

\section{Energy Storage}

Wireless sensor nodes typically have a duty-cycled operation: nodes 'sleep' for periods with a very low sleep current (typically around $1 \mu \mathrm{A}$ ), but draw a larger amount of current (around $30 \mathrm{~mA}$ ) for short times when the node is active and communicating. In order to deal with these occasional high current bursts, it is necessary to buffer harvested energy in a suitable storage device.

Early energy harvesting systems such as Heliomote [9] buffered energy in a rechargeable battery. Rechargeable batteries have a number of drawbacks - including memory effects and requirements for intricate monitoring when charging. More advanced systems such as Prometheus [4] use a battery in combination with a supercapacitor, which reduces stresses on the battery. However, this system has been developed for outdoor solar applications, where energy is plentiful. In the indoor energy harvesting scenario described earlier, it is unlikely that the optimal charging conditions for batteries will be achieved.

While NiMH batteries will accommodate low-current charging, the method of charge termination is non-trivial either $\mathrm{dT} / \mathrm{dt}$ or $-\mathrm{dV} / \mathrm{dt}$ are recommended, neither of which lend themselves to application with low-power energy harvesting. The voltage change due to charging is difficult to monitor for low charge currents, and heating effects are likely to be negligible and difficult to differentiate from environmental changes. Furthermore, NiMH batteries have a relatively high leakage current [3].
Lithium ion and lithium polymer batteries are also common. They require constant-current, followed by constant-voltage, charging (at a relatively high rate). In addition, during the constant-voltage charging phase their terminal voltage must be a relatively high $4.2 \mathrm{~V}$. They are not suitable for trickle charging, with overcharging possibly leading to hazardous reactions [3].

Supercapacitors are relatively insensitive to charge/discharge cycling, hence are more suitable than rechargeable batteries for low-level energy harvesting applications such as those described in this report. Supercapacitors exhibit similar behaviour characteristics to aluminium electrolytic capacitors, but have much higher capacitances (of the order of several Farads) [10].

It is for these reasons that supercapacitors are seen as the storage device of choice for micropower energy harvesting systems. While their leakage power is relatively high, this can be minimised by operating them at a low voltage (for example by operating two devices in series) and low temperature. Their lifetime can be extended by employing similar techniques. In addition, they obey the standard capacitor energy equation, which means that energy metering is straightforward.

\section{Management of Devices}

In view of the highly restricted energy levels obtained from energy harvesting devices, it follows that attention should be paid to ensuring efficient energy usage by the node's embedded software. Our prototype node adapts its duty cycle depending on the amount of harvested energy available, and conceals the complexity of interfacing with energy hardware by using an 'energy stack' architecture [2]. This modularises the interface between energy hardware and software, providing a common interface to the embedded software's application (top layer). The format of the energy stack is shown in Fig. 1.

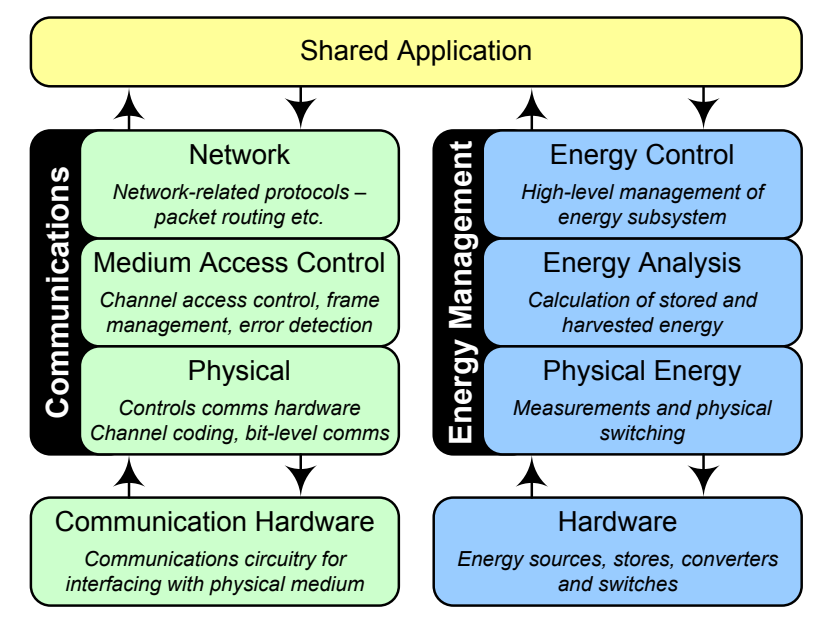

Fig. 1. Layout of the energy stack, shown next to the communication stack (adapted from [2])

Power priorities [11] are used to decide the node's activity level, and thresholds can be reconfigured while the system is running. The energy stack is a major strength of the solution, as it permits flexible adaptation of the 
energy subsystems to be supported by the embedded software with minimal effort.

The Energy Control layer presents the interface to the shared application layer. This interface consists of the power priority value of the system, and an interface to allow the thresholds to be changed. It also permits the application to 'see' the present energy status of the node, operating voltage, and allows the energy status value to be refreshed.

\begin{tabular}{|l|c|l|}
\hline Priority & Max\% & Description \\
\hline PP_Err & - & Error calculating status/unknown \\
\hline PP_Mains & - & Operating from the mains \\
\hline PP_5 & - & Plentiful energy \\
\hline PP_4 & 80 & \multirow{2}{*}{ Intermediate energy levels } \\
\hline PP_3 & 60 & \\
\hline PP_2 & 40 & \\
\hline PP_1 & 20 & Very limited energy \\
\hline PP_Empty & 2 & Cannot sustain transmission \\
\hline
\end{tabular}

Table 1 . Power priority values, as defined in the system

Table 1 shows the power priority values implemented in this system. The simplistic nature of the categories allows easy interpretation by the application layer. The application running on the node is shielded from the complex operations taking place in the energy stack: it is simply presented with this interface (but it can interrogate the stack for more detailed information if this is required).

\section{Power Status Monitoring}

A key issue in delivering energy-aware operation is in the effective determination of the power status of the node. Power status monitoring will ideally consider both the energy stored by the system, and the instantaneous power being generated by energy harvesting devices. While it may be a trivial task to estimate the amount of energy stored by a supercapacitor, for example, this is only of limited use as the system must also have an idea of the dynamics of power consumption with respect to supply voltage in order to reliably estimate the remaining lifetime. These issues are discussed in detail in [1].

Source monitoring is a more complex issue. Power conditioning circuits will expose harvesting devices to a load which is often undefined and varying. Furthermore, a complicating factor that the microcontroller, while engaged in taking the measurement, may also be operating directly or indirectly from the store or source being analysed. Ideally, energy sources would be isolated from their load during this analysis by means of a digital switch or transistor arrangement.

Implementation of this capability is likely to have an adverse impact on the performance of the power conditioning circuit. Power FET transistors have an onresistance that is typically from a few ohms to tens of ohms, effectively 'wasting' a proportion of the harvested energy. Careful design is essential, and a balance must be reached between the value of information obtained from energy sources, and the overall efficiency of the energy harvesting system.

\section{Complete System Integration}

By combining a number of energy sources on a sensor node, it is possible to enable the operation of the device over a wider range of conditions. Fig. 2 shows a simplified diagram of how the energy from a photovoltaic module and a vibration energy harvester are combined to power an autonomous sensor. It is shown that energy from both sources is collected in a common energy buffer, which directly powers the microcontroller, transceiver and sensing hardware.

A modified buck-boost converter has been developed, which permits the photovoltaic module to operate at a fixed voltage (set to its maximum power point voltage at the centre of the expected illumination range), regardless of the voltage of the energy buffer. Fig. 3 shows the charging curve from this optimized circuit compared to a simple diode, showing a 36\% time saving when coldstarting the system. The choice of harvesting device made by system designers will depend on the environmental energy available in each deployment scenario and the requirements of the autonomous sensor.

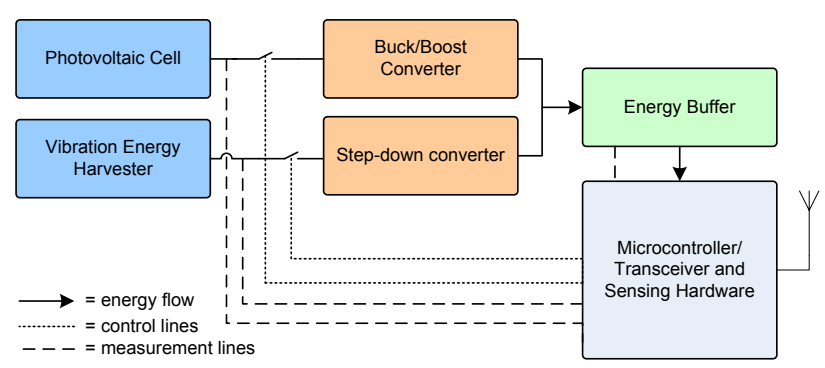

Fig. 2. Wireless autonomous sensor powered by photovoltaics and vibration energy harvesting

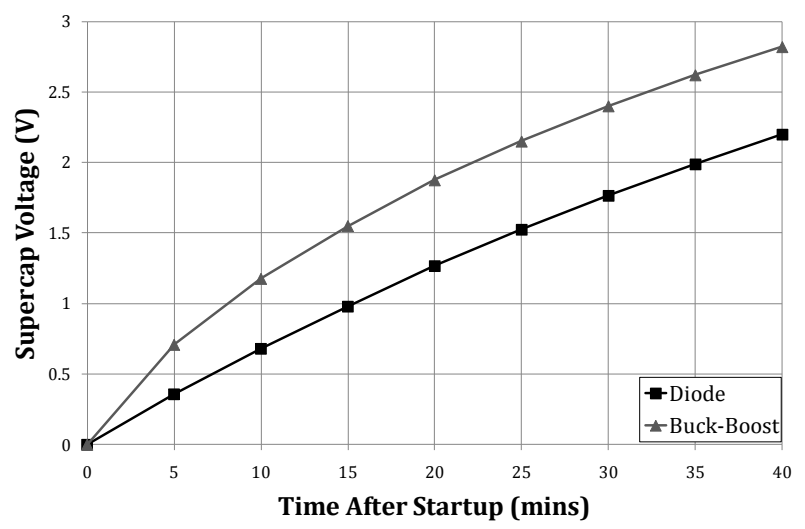

Fig. 3. Charging curves for $0.5 \mathrm{~F}$ supercapacitor from buck-boost converter and simple diode circuit

For the vibration energy harvester, which produces a half wave rectified output limited to $8 \mathrm{~V}$, a high-efficiency step-down switching regulator is used. This provides an efficient means of regulating the supply to that required by the rest of the node. To further increase the efficiency of the converter its output capacitor is allowed to charge up to correct operating levels prior to connection to the main supercapacitor-based energy store.

As shown in Fig. 2, the microcontroller can interrogate the open-circuit voltage of the photovoltaic module by 
disconnecting it from the buck/boost converter and measuring its voltage soon afterwards. From the opencircuit voltage, the system can estimate the nominal power being generated by the cell. In a similar way, the nominal output power from the vibration energy harvester can be estimated by shutting down the step-down converter and isolating its associated input capacitance. A known load is then switched in across the output of the vibration generator and the voltage across this load is measured to determine the instantaneous power level.

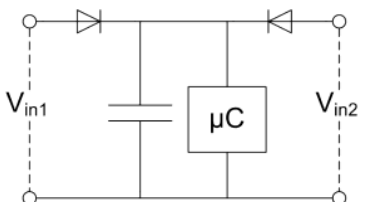

(a)

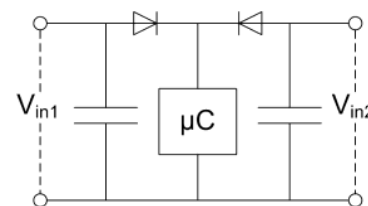

(b)
Fig. 4. Options for configuration of energy storage devices and energy sources for an energy-harvesting sensor node

Consideration needs to be given to the method of energy storage. There are two main options for combining separate energy harvesting devices on a single node. Fig. 4(a) shows a system where two energy sources act through diodes to charge a single supercapacitor adjacent to the microcontroller, and Fig. 4(b) shows a scheme where each energy source has a separate supercapacitor which feeds energy to the microcontroller via a diode.

The first option can potentially offer better long-term performance where both sources act to maintain a high voltage on the supercapacitor. The second option, while featuring a higher component count, may offer faster startup times as the capacitors can potentially be smaller only one of which is required to reach the microcontroller's turn-on voltage for the system to begin its operation.

In the example shown in Fig. 2, energy is stored in a common energy buffer (which consists of two $2.7 \mathrm{~V} 1 \mathrm{~F}$ supercapacitors in series, forming a total capacitance of $0.5 \mathrm{~F}$ and maximum voltage of $5.4 \mathrm{~V}$ ). The inputs from two energy harvesters are both driven through Schottky diodes to the common energy store, as shown in Fig. 4(a). In order to control and monitor the energy subsystem as shown in Fig. 2, two control lines and three ADC inputs on the microcontroller are used. This number could potentially be reduced by multiplexing the inputs to the microcontroller.

\section{Future Developments}

Further planned developments to the system are mainly related to the reconfigurability of the node to accommodate different energy harvesting (and storage) devices. It is planned that each energy-related device (e.g. storage unit, energy harvester with power conditioning circuit, etc.) will incorporate a simple memory device which can be interrogated by the sensor node on start-up. Information obtained from this would be used to set up parameters in the energy stack and permit the system to support the 'swapping' of energy hardware after programming, as presently any changes in the hardware must be hard-coded into the embedded software.

\section{Conclusions}

This report has outlined the issues related to powering sensor nodes from conventional energy sources and harvested energy. A hardware and embedded software scheme for managing energy harvested from multiple sources has been presented, and applied to a system generating energy from both light and vibration. Further developments to the system have also been considered.

\section{Acknowledgments}

The work reported on in this paper was undertaken as part of the Data Information Fusion Defence Technology Centre (DIF DTC) Phase II 'Adaptive Energy-Aware Sensor Networks' project, funded jointly by the UK Ministry of Defence and General Dynamics UK.

\section{References}

1. Weddell, A. S. et al, "Alternative Energy Sources for Sensor Nodes: Rationalized Design for Long-Term Deployment," Proc 25 $5^{\text {th }}$ Intl Instrumentation and Measurement Technology Conf, Victoria, Canada, May 2008, pp. 1370-1375.

2. Merrett, G. V. et al, "A Structured Hardware/Software Architecture for Embedded Sensor Nodes," Proc $17^{\text {th }}$ Intl Conf on Computer Communications and Networks, St Thomas, VI, Aug. 2008 (in press).

3. Linden, D., Handbook of Batteries, McGraw-Hill (New York, 2002).

4. Jiang, X. et al, "Perpetual Environmentally Powered Sensor Networks," Proc $4^{\text {th }}$ Intl Symposium on Information Processing in Sensor Networks, Los Angeles, CA, Apr. 2005, pp. 463-468.

5. George, S., "Development of a Vibration-Powered Wireless Temperature Sensor and Accelerometer for Health Monitoring," Proc 2006 IEEE Aerospace Conf, Big Sky, MT, Mar. 2006, 8 pp.

6. Koukharenko, E. et al, "Development of nanostructures for thermoelecric microgenerators using ion-track lithography," Electronics Letters, Vol. 44, No. 7 (2008), pp. 500-501.

7. Parker, J., "Perpetuum Vibration Energy HarvesterPowered Wireless Condition Monitoring," Application Note, Perpetuum Ltd, Southampton, UK, Apr. 2007.

8. "TE-Power-One Thermogenerator Evaluation Kits Based on Micropelt MPG Series," Datasheet, Micropelt GmbH, Freiburg, Germany, Apr. 2008.

9. Raghunathan, V. et al, "Design considerations for solar energy harvesting wireless embedded systems," Proc $4^{\text {th }}$ Intl Symp on Info Processing in Sensor Networks, Los Angeles, CA, Apr. 2005, pp. 457-462.

10. "Gold Capacitors Technical Guide," Technical Guide, Panasonic Industrial Co., Secausus, NJ, May 2005.

11. Merrett, G. et al, "Energy Managed Reporting for Wireless Sensor Networks," Sensors and Actuators A: Physical, Vol. 142, No. 1 (2008), pp. 379-389. 